\title{
Characterization of Carbon Nanofibers Treated with Thermal Nitrogen as a Catalyst Support Using Point-of-Zero Charge Analysis
}

\author{
Thien Duc Nguyen Van, ${ }^{1}$ Suriati Sufian, ${ }^{1}$ Nurlidia Mansor, ${ }^{1}$ and Noorhana Yahya ${ }^{2}$ \\ ${ }^{1}$ Chemical Engineering Department, Universiti Teknologi Petronas, 31750 Tronoh, Perak Darul Ridzuan, Malaysia \\ ${ }^{2}$ Fundamental and Applied Science Department, Universiti Teknologi Petronas, 31750 Tronoh, Perak Darul Ridzuan, Malaysia \\ Correspondence should be addressed to Thien Duc Nguyen Van; nvthienduc84@gmail.com
}

Received 5 July 2014; Accepted 27 August 2014; Published 14 September 2014

Academic Editor: Yongchun Hong

Copyright (C) 2014 Thien Duc Nguyen Van et al. This is an open access article distributed under the Creative Commons Attribution License, which permits unrestricted use, distribution, and reproduction in any medium, provided the original work is properly cited.

\begin{abstract}
The chemical and physical purification of carbon nanofiber exposes more anchoring sites between meal precursors and carbon surface but thermal $\mathrm{N}_{2}$ gas flow maintains the crystal's structure as well as its defect and edge sites, referred to as active sites or anchoring sites. After calcination in nitrogen at $450^{\circ} \mathrm{C}$, samples were characterized by Raman spectra X-ray diffraction, as well as thermogravimetric and nitrogen physisorption analyses. Results showed a relatively lower fraction of amorphous carbon to graphite, indicating a greater removal of amorphous carbon. Moreover, the disorder intensity of carbon nanofibers that were treated in $\mathrm{N}_{2}$ flow rate of $1 \mathrm{~L} / \mathrm{min}$ and 3 hours, called $1 \mathrm{Gcom}-3 \mathrm{~h}$ sample, achieved far more defect sites compared with unmodified carbon nanofiber. In addition, the surface areas of mesoporous carbon nanofibers decreased over prolonged residence time. The carbon nanofiber support-metal cation interaction therefore improved the deposition of iron when the point-of-zero charge reading was greater than four.
\end{abstract}

\section{Introduction}

A heterogeneous catalyst should possess active sites on its solid surface as a key property. In order to maximize the number of active sites, researchers typically distribute active sites on carbon supports rather than these on oxidic supports because of carbon surface inertness property in activity and selectivity of any chemical reactions [1]. Carbon nanofiber (CNF) which is one kind of carbon support has unique advantageous properties including chemical inertia as well as electrical and thermal stability $[1,2]$. Carbon's hydrogen adsorption and activation may be the reason for its catalytic activity [3].

It is different from CNF structure, morphology, and chemistry surface that impact on metallic particles deposition [4-6]. CNFs consisting of strong tangled agglomeration structures establish an open pore volume and mesopore structure which reduce diffusion limited transfers that have a key role in heterogeneous catalysis [7]. CNF aggregates also display a high surface area of $100-250 \mathrm{~m}^{2} / \mathrm{g}$. As synthesized, CNF exists in types of impurities (amorphous carbon) and graphite (graphene and defect structure) as well as metal based catalyst (metal carbide). Most scientists have successfully explored to modify graphite structure and remove amorphous carbon and metal carbide. They used refluxing acid, peroxide, or oxygen thermal treatment to attach more functional groups which become active sites or anchoring centers for the metal precursors [8-17], at preferred defect sites. Also, Mawhinney et al., 2000, suggested $\mathrm{O}_{3}$ method for titrating surface defect site density on carbon nanofilaments [18]. Barranco et al., 2010, and Sadezky et al., 2005, suggested that Raman analysis can determine the degree of graphitization or defect $\left(I_{\mathrm{G}} / I_{\mathrm{D}}+I_{\mathrm{G}}\right.$ or $I_{\mathrm{D}} / I_{\mathrm{D}}+I_{\mathrm{G}}$, resp.) from CNF surface $[19,20]$.

Furthermore, there are many types of morphology as fishbone (f-), platelet ( $\mathrm{p}-)$, ribbon ( $\mathrm{r}-)$, and irregular CNF but fishbone and platelet CNF have high edge sites that are good position for adsorption or modification CNF structure 
surface [21]. Ochoa-Fernández et al. in 2005 said that f-CNF strongly influences metallic catalyst deposition more than other morphologies because of more curvature [22]. Based on treatments above, CNF structures of different morphology types such as f-CNFs, p-CNFs, r-CNFs, and irregular CNFs [23-26] may become damaged and pose difficulty when attempting to control the number of active sites. This, in turn, limits electron transfer between active sites of metal catalyst. Also, point-of-zero charge (PZC) analysis plays an important role in the evaluation of surface chemistry support as it influences the predictability of interactions between metal ions and the carbon support structure in suspended solutions of varied $\mathrm{pH}$. Therefore, the interaction of CNF and deposited metal ion depends on the anion and cation balance [27] whilst carbon surface modification techniques have become essential for efficient deposition of metal precursors. However, using nitrogen treatment is different from common approaches using chemical reacting such as $\mathrm{KMnO}_{4}, \mathrm{H}_{2} \mathrm{O}_{2}$, and $\mathrm{HNO}_{3}$ oxidation. It has been limited in modifying $\mathrm{CNF}$ surface structure.

This research utilized calcination in nitrogen gas flow to detach acidic and basic groups from the surface of assynthesized CNF and remove the amorphous carbon phase because functional oxygen groups and the amorphous carbon phase inhibit the supported catalyst during nitrogen hydrogenation $[28,29]$. As negative charges on the support surface affect interactions between iron colloids and the CNF support, the aim of this research was to explore CNF purification with thermal $\mathrm{N}_{2}$ gas flow and CNF surface properties for purposes of supported catalyst preparation.

\section{Materials and Methods}

CNFs were purchased from Carbon Nanomaterial Co., LTD., and modified in a fixed-bed alumina reactor. Treatments were carried out under different conditions of residence time $(1,3$, and $6 \mathrm{~h})$ and nitrogen flow rate $(0.5$ and $1 \mathrm{~L} / \mathrm{min})$ at $450^{\circ} \mathrm{C}$ because $\mathrm{CNF}$ decomposes above $500^{\circ} \mathrm{C}$ (Figure 1 ). Degradation temperatures were determined by Pyris 1 thermogravimetric analysis (TGA) using the same gas at a heating rate of $20^{\circ} \mathrm{C} / \mathrm{min}$. Also, for instance, name code of sample is $1 \mathrm{Gcom}-1 \mathrm{~h}$. It means that 1 is symbol of $1 \mathrm{~L} / \mathrm{min}$; Gcom is name of CNF; and $1 \mathrm{~h}$ is calcination time.

Samples were prepared in isopropanol under sonication and resultant structures were observed under field emission transmission electron microscopy (FETEM; Zeiss Libra @200) for all CNF samples. Surface area and pore size ranges were analyzed by nitrogen ASAP 2020 physisorption where samples were degassed at $250^{\circ} \mathrm{C}$ over four hours prior to analysis. The degree of graphitization of samples and their relative amorphous values were assessed by (i) obtaining the Raman spectra with a Horiba HR 800 spectrometer operating at $514.53 \mathrm{~nm}$ at room temperature and (ii) Bruker AXS D8advance $\mathrm{X}$-ray diffraction (XRD) using copper $\mathrm{K} \alpha$ radiation (wavelength, $\lambda=0.154 \mathrm{~nm}$ ) and 2 theta from $2-80^{\circ}$. The zeta potential for CNFs as a function of $\mathrm{pH} 2-10$ was also tested with a Malvern Nano-ZS Zetasizer (DKSH Technology $\mathrm{Sdn}$. Bhd.) in solutions of $0.01 \mathrm{M} \mathrm{NaCl}$ mixed with carbon

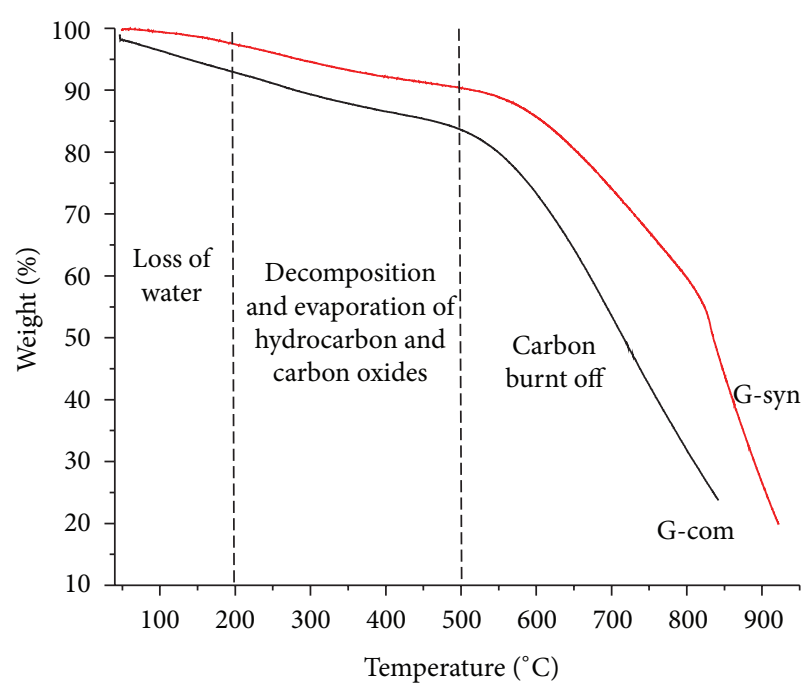

FIgURE 1: Degradation behavior of CNF from TGA spectra.

TABLE 1: X-Ray diffraction intensity of carbon nanofiber.

\begin{tabular}{lccc}
\hline Sample name & $\begin{array}{c}\text { Calcinated } \\
\text { conditions at } \\
450^{\circ} \mathrm{C}\end{array}$ & $\begin{array}{c}\text { Intensity } \\
\text { (counts) }\end{array}$ & $d_{002}(\mathrm{~nm})$ \\
\hline G-com & - & 21.0 & 0.344 \\
1Gcom-1h & $1 \mathrm{~L} / \mathrm{min}, 1 \mathrm{~h}$ & 21.0 & 0.343 \\
1Gcom-3h & $1 \mathrm{~L} / \mathrm{min}, 3 \mathrm{~h}$ & 25.0 & 0.343 \\
1 Gcom-6h & $1 \mathrm{~L} / \mathrm{min}, 6 \mathrm{~h}$ & 23.7 & 0.343 \\
$0.5 \mathrm{Gcom}-3 \mathrm{~h}$ & $0.5 \mathrm{~L} / \mathrm{min}, 3 \mathrm{~h}$ & 25.4 & 0.343 \\
\hline
\end{tabular}

nanofiber powder with either $0.5 \mathrm{M} \mathrm{NaOH}$ or $0.5 \mathrm{M} \mathrm{HCl}$ to alter $\mathrm{pH}[30]$.

\section{Results and Discussion}

Commercial carbon nanofiber (G-com) and as-synthesized carbon nanofiber (G-syn) [23] weight losses are shown in Figure 1. Changes were rapid at the beginning $\left(500^{\circ} \mathrm{C}\right)$. Gcom had one inclination at $550^{\circ} \mathrm{C}$ but G-syn had two inclination change stages $\left(600\right.$ and $\left.800^{\circ} \mathrm{C}\right)$. Overall, the degradation level of G-com was higher than that of G-syn because of Gsyn's greater structural stability. The TGA curve shows three regions with the first between 50 and $200^{\circ} \mathrm{C}$ due to the loss of water. The second $\left(200-500^{\circ} \mathrm{C}\right)$ shows a decomposition of light amorphous polycarbons from carbon dioxide that are easily vaporized [31]. The final region indicates the gradual destruction of the CNF's structure above $500^{\circ} \mathrm{C}$ for both Gcom and G-syn. All observed changes were due to amorphous carbon layers and differences in morphological structure $[32,33]$. Thus, the purpose of thermal treatment in an inert environment is to eliminate amorphous carbon layers and increase defective sites on the carbon surface (as shown in Table 1). This may enhance the ability of metal adsorption on CNF surfaces. As discussed, G-com and G-syn samples were calcinated at $450^{\circ} \mathrm{C}$ to avoid CNF structural breakdown; however, CNF surface defects remained. 
TABLE 2: Graphite nanofiber peak positions and the intensity ratio of Raman spectroscopy bands.

\begin{tabular}{|c|c|c|c|c|c|}
\hline Sample name & $I_{\mathrm{D}}(\%) 1300-1400 \mathrm{~cm}^{-1}$ & $I_{\mathrm{G}}(\%) 1580-1600 \mathrm{~cm}^{-1}$ & $\begin{array}{c}\text { Degree of } \\
\text { graphitization }\end{array}$ & $I_{\mathrm{A}}(\%)$ & $\begin{array}{c}\text { The relative value ratio } \\
\text { of amorphous carbon to } \\
\text { graphite intensity }\end{array}$ \\
\hline G-com & 180 & 150 & 0.454 & 65 & 0.433 \\
\hline $1 \mathrm{Gcom}-1 \mathrm{~h}$ & 135 & 95 & 0.413 & 45 & 0.473 \\
\hline $1 \mathrm{Gcom}-3 \mathrm{~h}$ & 110 & 85 & 0.435 & 35 & 0.411 \\
\hline 1 Gcom-6h & 95 & 67 & 0.413 & 30 & 0.448 \\
\hline $0.5 \mathrm{Gcom}-3 \mathrm{~h}$ & 115 & 85 & 0.425 & 40 & 0.471 \\
\hline
\end{tabular}

$I_{\mathrm{D}}, I_{\mathrm{G}}, I_{\mathrm{A}}$ : disorder, graphite, and amorphous intensities, respectively. D-band and G-band: disorder $\left(\mathrm{C}_{\mathrm{sp}}{ }^{3}\right)$ and graphite $\left(\mathrm{C}_{\mathrm{sp}}{ }^{2}\right)$ frequency.

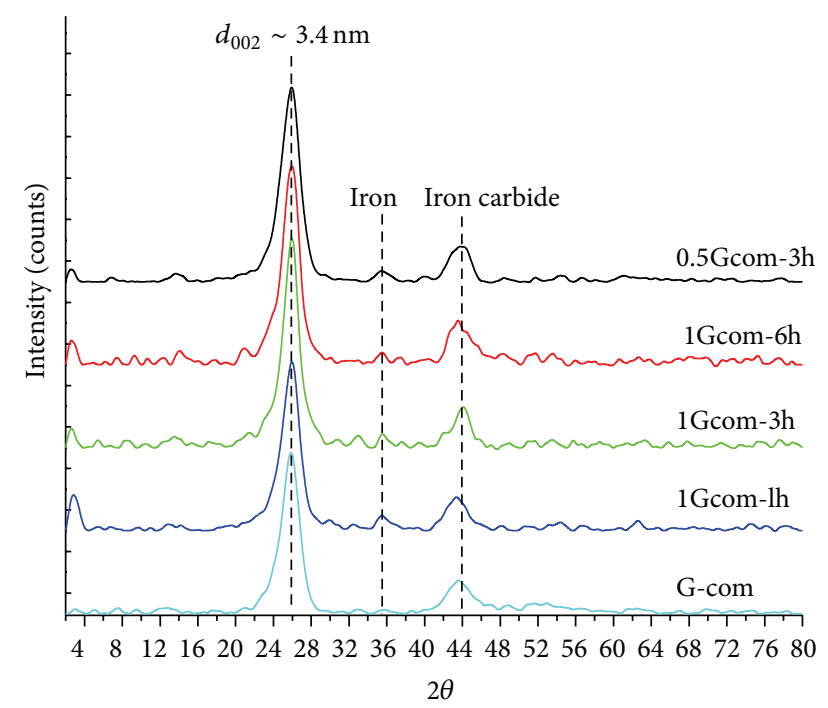

FIgURE 2: X-ray diffraction results for CNFs and modified CNFs.

Table 1 illustrates how the X-ray diffraction intensity was amplified over one to three hours (1Gcom-1h; $1 \mathrm{Gcom}-$ $3 \mathrm{~h})$. However, intensity changed slightly as residence time increased to six hours (1Gcom-6h) and also when a reduction in flow rate to $500 \mathrm{~mL} / \mathrm{min}(0.5 \mathrm{Gcom}-3 \mathrm{~h})$ occurred. These findings were similar to Raman's results for degrees of graphitization (see Table 2) as discussed below.

The CNF XRD pattern in Figure 2 shows more information of d-spacing interlayer of graphite and existence elements in samples. The data of iron, iron carbide, and carbon nanofiber were matched with reference data of 130534, 35-0772, and 75-2078 based on JCPDS, respectively. Figure 2 displays that no change occurred in the d-spacing interlayer of crystalline graphite in $2 \theta=20-30^{\circ}$. The iron carbide changes did occur in $2 \theta=40-48^{\circ}$ [23]. The modified samples $(1 \mathrm{Gcom}-1 \mathrm{~h}, 1 \mathrm{Gcom}-3 \mathrm{~h}, 1 \mathrm{Gcom}-6 \mathrm{~h}$, and $0.5 \mathrm{Gcom}-$ $3 \mathrm{~h}$ ) each displayed iron peaks between 30 and $40^{\circ}$ that were not present in the G-com sample [34]. This indicated that treatment abolished amorphous carbon layers and revealed encapsulated iron.

We used Raman spectra analysis to explore intrinsic defects in the graphite lattice that were responsible for the symmetrical breakdown of the entire crystalline network [35]. Raman spectra of microcrystalline graphite showed a disordered band (D peak) between 1300 and $1400 \mathrm{~cm}^{-1}$ due to lattice defects and a loss of long-range in-plane symmetry. However, a G-band peak $\left(1580-1600 \mathrm{~cm}^{-1}\right)$ appeared due to in-plane bond stretching of carbon atom $\mathrm{sp}^{2}$ hybridization. Also, the intensity of the D peak (Table 2) was higher than that of the G peak, implying high disordered graphitic structures [20] and corrugation of the CNF [36]. The degree of graphitization was estimated by the intensity ratio: $I_{\mathrm{G}} /\left(I_{\mathrm{D}}+\right.$ $\left.I_{\mathrm{G}}\right)$ [37] or else $1-I_{\mathrm{G}} /\left(I_{\mathrm{D}}+I_{\mathrm{G}}\right)$ is for defect degree. In addition, increased CNF graphitization results agreed with the sample (1Gcom-3h) because unstable carbon structures were removed via calcination. These findings also endorsed our XRD results as shown in Table 1, which, taken together, indicated that the majority of amorphous carbon was gradually eliminated. In Table 2 the graphitization degree value of modified samples decreases. It means that using N2 thermal treatment can improve the defect surface structure of CNF because of the defect or disordered degree value of modified samples increases.

We determined that the CNF's amorphous phase occurred at Raman shift $1550 \mathrm{~cm}^{-1}$ [35]. Table 2 describes results indicating that thermal treatment enhanced the CNF by the incineration of amorphous carbon. The relative value of the amorphous carbon filament was estimated by the integrated intensity ratio of $I_{\mathrm{A}} / I_{\mathrm{G}}$ [9], which decreased as residence time increased to $3 \mathrm{~h}$ with a nitrogen flow rate of $1 \mathrm{~L}$ per min (1Gcom-3h). However, the amorphous phase increased with diminishing nitrogen flow $(500 \mathrm{~mL}$ per min) $(0.5 \mathrm{Gcom}-3 \mathrm{~h})$. The relative value of amorphous carbon was lowest for sample $1 \mathrm{Gcom}-3 \mathrm{~h}$ as shown in Figure 1. Amorphous carbon reflected a weight loss of approximately $15 \%$ (G-com) below $500^{\circ} \mathrm{C}$. By reducing amorphous carbon, $\mathrm{CNF}$ pore size increased and could then be characterized as mesopores (Table 3 ) that allowed an easier internal diffusion of metal ions. Nevertheless, the defective surface of the CNF remained constant which also improved the adsorption of carbon filaments.

Hence, it appeared that nitrogen physisorption (Table 3) established mesopores with diameters $>2 \mathrm{~nm}$ [38]. With thermal $\mathrm{N}_{2}$ flow rates of $500 \mathrm{~mL} / \mathrm{min}$ and $1000 \mathrm{~mL} / \mathrm{min}$ and over residence intervals of 1,3 , and 6 hours, both surface area and pore size decreased compared to nonmodified samples (Gcom) due to amorphous phase removal of CNF and increased rearrangement of the graphite structure. In addition, there was a wide distribution of pore width ranging from 1.8 to $1415.4 \mathrm{~nm}$. The range of pore size for modified samples was 
TABLE 3: Surface area and pore size of CNF.

\begin{tabular}{lccc}
\hline Sample name & Pore size $(\mathrm{nm})$ by BJH & Surface area $\left(\mathrm{m}^{2} / \mathrm{g}\right)$ by BET & Pore width range $(\mathrm{nm})$ \\
\hline G-com & 17.627 & 114.485 & $2 .-267.3$ \\
1Gcom-1h & 16.175 & 110.119 & $2.7-1226.5$ \\
1Gcom-3h & 15.829 & 90.238 & $1.9-382.7$ \\
1Gcom-6h & 15.627 & 97.731 & $1.9-1415.4$ \\
0.5 Gcom-3h & 17.757 & 95.011 & $1.8-211.9$ \\
\hline
\end{tabular}

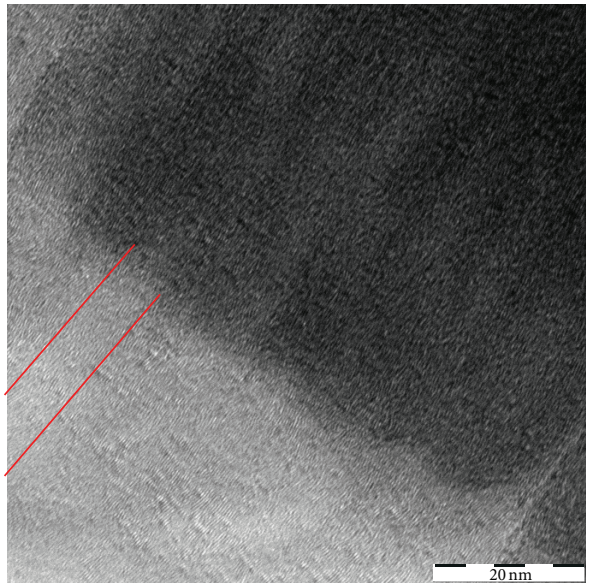

(a)

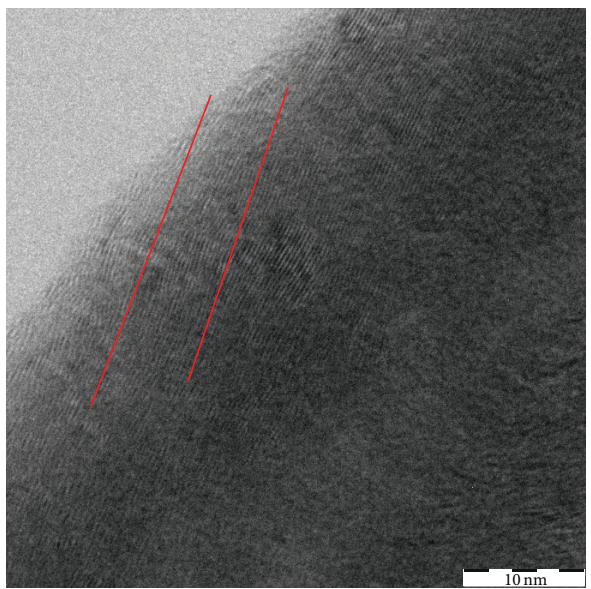

(b)

Figure 3: CNF TEM images: p-CNF, 3(a); f-CNF, 3(b).

larger than that of G-com, indicating that the majority of pores allowed for more efficient metal ion adsorption on deposition.

Typical CNF TEM images of two types are shown in Figure 3 based on angles between graphene layers and the growth axis. CNF graphene layers called platelet CNFs ( $\mathrm{p}-$ $\mathrm{CNF}$ ) are arranged perpendicular to the axis (see Figure 3(a)). The G-com structure showed angles of approximately $30^{\circ}$ between graphene layers and the $\mathrm{CNF}$ axis, also known as fishbone CNF (f-CNF), seen in Figure 3(b). This phenomenon indicated the existence of more edge sites on $\mathrm{p}$ $\mathrm{CNF}$ and $\mathrm{f}-\mathrm{CNF}$ structures, in addition to defect sites as active

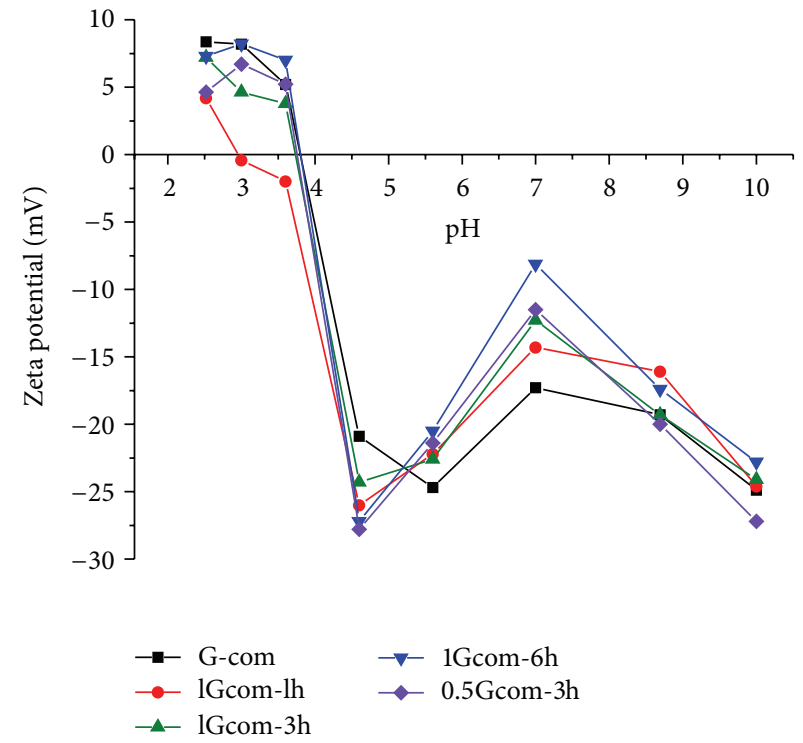

FIGURE 4: CNF zeta potential (PZC) under different treatment conditions.

sites, all of which aid metal ion adsorption on the CNF surface $[39,40]$.

CNF zeta potential characterization is shown in Figure 4. Varied relative point-of-zero charges (PZC) for $\mathrm{p}$-CNF and f-CNF ranged between $\mathrm{pH} 3$ and 4 and were lower than those for argon gas modified CNFs as reported by Zhu et al. [30], most likely due to extant iron within the CNF. Except for the $1 \mathrm{Gcom}-1 \mathrm{~h}$ sample, PZC for all other CNF samples varied between $\mathrm{pH} 3.5$ and 4. Vinke et al. [41] and Solar et al. [42] suggested that the PZC of carbon was subject to total surface oxygen and the nature and distribution of surface oxygen sites. These results therefore implied that oxygen was removed by heat treatment.

Furthermore, the PZC value aids our understanding of changes that occur in surface charges of the CNF support using impregnation of the supported catalyst preparation and consequently also for the dispersion of the metallic active phase as discussed by Regalbuto [43]. An electrostatic attraction between the CNF support and cation salt depends on the $\mathrm{pH}$ medium as formulated over or under $\mathrm{PZC}$ value. Figure 4 shows PZC values for a basic suspension $(\mathrm{pH}>$ $4)$ and for an acid medium $(\mathrm{pH}<3)$. Rodríguez-Reinoso 1998 similarly reviewed that Pt complex salt can adsorb on surface of oxygen-free carbon by electrostatic force [1] and it belonged to precursor salt ion in acid or basic solution. 
Therefore, a deposition method can be developed for $\mathrm{pH}$ values greater than the CNF support's PZC since cation salts can interact with negative charges of surface carbons. Also, the larger the interaction, the higher the potential difference between the CNF support and metal ion [4, 30, 44]. This approach controls both metal loading and particle size for interaction and adsorption characteristics during the process of deposition.

\section{Conclusion}

Using thermal purification in nitrogen gas, $\mathrm{p}-\mathrm{CNF}$ and $\mathrm{f}-\mathrm{CNF}$ structures were broken down to release impurities during the amorphous phase and still retain iron metal and defect sites. Amorphous carbon layers were efficiently eliminated to minimize the ratio of amorphous carbon to graphite. Furthermore, CNF defect structures increase after modification and get minimum amorphous carbon with sample of 1 Gcom-3h. The surface area of the sample was $90.238 \mathrm{~m}^{2} / \mathrm{g}$, and pore width ranged from micro- to macropore. These results clearly indicated easier metal ion diffusion. Finally, electrostatic attraction between negative CNF support and cation salt improves when the $\mathrm{pH}$ medium is greater than a PZC value of four.

\section{Conflict of Interests}

The authors declare that there is no conflict of interests regarding the publication of this paper.

\section{Acknowledgments}

The authors gratefully acknowledge MOE for its financial support (LRGS-One Baja Funder and FRGS), Universiti Teknologi PETRONAS for its generous Graduate Assistantship scheme and lab facilities, and also Mr. Albert Lim and Mr. Simon Choo from DKSH Technology Sdn. Bhd. for supporting this research with the Malvern Nano-ZS Zetasizer equipment.

\section{References}

[1] F. Rodríguez-Reinoso, "The role of carbon materials in heterogeneous catalysis," Carbon, vol. 36, no. 3, pp. 159-175, 1998.

[2] P. Sep and J. L. Figueiredo, Carbon Nanomaterials for Catalysis, John Wiley \& Sons, New York, NY, USA, 2009.

[3] E. Furimsky, Carbons and Carbon Supported Catalysts in Hydroprocessing, The Royal Society of Chemistry, Cambridge, UK, 2008.

[4] I. Kvande, S. T. Briskeby, M. Tsypkin et al., "On the preparation methods for carbon nanofiber-supported Pt catalysts," Topics in Catalysis, vol. 45, no. 1-4, pp. 81-85, 2007.

[5] M. A. Fraga, E. Jordão, M. J. Mendes, M. M. A. Freitas, J. L. Faria, and J. L. Figueiredo, "Properties of carbon-supported platinum catalysts: role of carbon surface sites," Journal of Catalysis, vol. 209, no. 2, pp. 355-364, 2002.

[6] W. Raróg-Pilecka, E. Miśkiewicz, M. Matyszek, Z. Kaszkur, L. Kępiński, and Z. Kowalczyk, "Carbon-supported cobalt catalyst for ammonia synthesis: effect of preparation procedure," Journal of Catalysis, vol. 237, no. 1, pp. 207-210, 2006.

[7] K. P. de Jong, "Synthesis of supported catalysts," Current Opinion in Solid State and Materials Science, vol. 4, no. 1, pp. 55-62, 1999.

[8] J. L. Figueiredo, M. F. R. Pereira, M. M. A. Freitas, and J. J. M. Órfão, "Modification of the surface chemistry of activated carbons," Carbon, vol. 37, no. 9, pp. 1379-1389, 1999.

[9] J. Li, M. J. Vergne, E. D. Mowles, W.-H. Zhong, D. M. Hercules, and C. M. Lukehart, "Surface functionalization and characterization of graphitic carbon nanofibers (GCNFs)," Carbon, vol. 43, no. 14, pp. 2883-2893, 2005.

[10] S. Lim, A. Shimizu, S.-H. Yoon, Y. Korai, and I. Mochida, "High yield preparation of tubular carbon nanofibers over supported Co-Mo catalysts," Carbon, vol. 42, no. 7, pp. 1279-1283, 2004.

[11] J. S. Noh and J. A. Schwarz, "Effect of $\mathrm{HNO}_{3}$ treatment on the surface acidity of activated carbons," Carbon, vol. 28, no. 5, pp. 675-682, 1990.

[12] R. A. Ross, C. Fairbridge, and J. R. MacCallum, "Carbon fibres as supports for transition metal catalysts in hydrocarbon oxidation reactions," Carbon, vol. 23, no. 2, pp. 209-213, 1985.

[13] J.-F. Colomer, P. Piedigrosso, A. Fonseca, and J. B. Nagy, "Different purification methods of carbon nanotubes produced by catalytic synthesis," Synthetic Metals, vol. 103, no. 1-3, pp. 2482-2483, 1999.

[14] W.-K. Choi, S.-G. Park, H. Takahashi, and T.-H. Cho, "Purification of carbon nanofibers with hydrogen peroxide," Synthetic Metals, vol. 139, no. 1, pp. 39-42, 2003.

[15] T. Jeong, W.-Y. Kim, and Y.-B. Hahn, "A new purification method of single-wall carbon nanotubes using $\mathrm{H}_{2} \mathrm{~S}$ and $\mathrm{O}_{2}$ mixture gas," Chemical Physics Letters, vol. 344, no. 1-2, pp. 1822, 2001.

[16] K. B. Shelimov, R. O. Esenaliev, A. G. Rinzler, C. B. Huffman, and R. E. Smalley, "Purification of single-wall carbon nanotubes by ultrasonically assisted filtration," Chemical Physics Letters, vol. 282, no. 5-6, pp. 429-434, 1998.

[17] M. L. Toebes, F. F. Prinsloo, J. H. Bitter, A. J. van Dillen, and K. P. de Jong, "Influence of oxygen-containing surface groups on the activity and selectivity of carbon nanofiber-supported ruthenium catalysts in the hydrogenation of cinnamaldehyde," Journal of Catalysis, vol. 214, no. 1, pp. 78-87, 2003.

[18] D. B. Mawhinney, V. Naumenko, A. Kuznetsova, J. T. Yates Jr., J. Liu, and R. E. Smalley, "Surface defect site density on single walled carbon nanotubes by titration," Chemical Physics Letters, vol. 324, no. 1-3, pp. 213-216, 2000.

[19] V. Barranco, M. A. Lillo-Rodenas, A. Linares-Solano et al., "Amorphous carbon nanofibers and their activated carbon nanofibers as supercapacitor electrodes," Journal of Physical Chemistry C, vol. 114, no. 22, pp. 10302-10307, 2010.

[20] A. Sadezky, H. Muckenhuber, H. Grothe, R. Niessner, and U. Pöschl, "Raman microspectroscopy of soot and related carbonaceous materials: spectral analysis and structural information," Carbon, vol. 43, no. 8, pp. 1731-1742, 2005.

[21] P. Serp and J. L. Figueiredo, Carbon Materials for Catalysis, John Wiley \& Sons, 2009.

[22] E. Ochoa-Fernández, D. Chen, Z. Yu, B. Tøtdal, M. Rønning, and A. Holmen, "Carbon nanofiber supported Ni catalyst: effects of nanostructure of supports and catalyst preparation," Catalysis Today, vol. 102-103, pp. 45-49, 2005.

[23] S. Sufian, "Irregular configurations of carbon nanofibers," in Carbon and Oxide Nanostructures, vol. 5, pp. 125-141, Springer, Berlin, Germany, 2011. 
[24] W. B. Downs and R. T. K. Baker, "Novel carbon fiber-carbon filament structures," Carbon, vol. 29, no. 8, pp. 1173-1179, 1991.

[25] X. Jian, M. Jiang, Z. Zhou et al., "Preparation of high purity helical carbon nanofibers by the catalytic decomposition of acetylene and their growth mechanism," Carbon, vol. 48, no. 15, pp. 4535-4541, 2010.

[26] R. T. Yang and J. P. Chen, "Mechanism of carbon filament growth on metal catalysts," Journal of Catalysis, vol. 115, no. 1, pp. 52-64, 1989.

[27] E. Cristiano, Y.-J. Hu, M. Siegfried, D. Kaplan, and H. Nitsche, "A comparison of point of zero charge measurement methodology," Clays and Clay Minerals, vol. 59, no. 2, pp. 107-115, 2011.

[28] Z. Li, C. Liang, Z. Feng, P. Ying, D. Wang, and C. Li, "Ammonia synthesis on graphitic-nanofilament supported Ru catalysts," Journal of Molecular Catalysis A: Chemical, vol. 211, no. 1-2, pp. 103-109, 2004.

[29] C. H. Bartholomew, "Mechanisms of catalyst deactivation," Applied Catalysis A: General, vol. 212, no. 1-2, pp. 17-60, 2001.

[30] J. Zhu, T. Zhao, I. Kvande, D. Chen, X. Zhou, and W. Yuan, "Carbon nanofiber-supported Pd catalysts for heck reaction: effects of support interaction," Chinese Journal of Catalysis, vol. 29, no. 11, pp. 1145-1151, 2008.

[31] T. G. Ros, A. J. van Dillen, J. W. Geus, and D. C. Koningsberger, "Surface structure of untreated parallel and fishbone carbon nanofibres: an infrared study," ChemPhysChem, vol. 3, no. 2, pp. 209-214, 2002.

[32] A. B. Dongil, B. Bachiller-Baeza, A. Guerrero-Ruiz, I. Rodríguez-Ramos, A. Martínez-Alonso, and J. M. D. Tascón, "Surface chemical modifications induced on high surface area graphite and carbon nanofibers using different oxidation and functionalization treatments," Journal of Colloid and Interface Science, vol. 355, no. 1, pp. 179-189, 2011.

[33] N. J. Welham and J. S. Williams, "Extended milling of graphite and activated carbon," Carbon, vol. 36, no. 9, pp. 1309-1315, 1998.

[34] M.-S. Wu, Y.-H. Ou, and Y.-P. Lin, "Electrodeposition of iron oxide nanorods on carbon nanofiber scaffolds as an anode material for lithium-ion batteries," Electrochimica Acta, vol. 55, no. 9, pp. 3240-3244, 2010.

[35] T. V. Reshetenko, L. B. Avdeeva, Z. R. Ismagilov et al., "Catalytic filamentous carbon: structural and textural properties," Carbon, vol. 41, no. 8, pp. 1605-1615, 2003.

[36] T. W. Ebbesen, Carbon Nanotubes: Preparation and Properties, CRC, 1996.

[37] J.-H. Ting, C.-C. Chang, S.-L. Chen, D.-S. Lu, C.-Y. Kung, and F.-Y. Huang, "Optimization of field emission properties of carbon nanotubes by Taguchi method," Thin Solid Films, vol. 496, no. 2, pp. 299-305, 2006.

[38] J. B. Condon, Surface Area and Porosity Determinations by Physisorption: Measurements and Theory, Elsevier, New York, NY, USA, 2006.

[39] C. Park and R. T. K. Baker, "Catalytic behavior of graphite nanofiber supported nickel particles. 3 . The effect of chemical blocking on the performance of the system," The Journal of Physical Chemistry B, vol. 103, no. 13, pp. 2453-2459, 1999.

[40] F. Rodríguez-Reinoso and M. Molina-Sabio, "Textural and chemical characterization of microporous carbons," Advances in Colloid and Interface Science, vol. 76-77, pp. 271-294, 1998.

[41] P. Vinke, M. van der Eijk, M. Verbree, A. F. Voskamp, and H. van Bekkum, "Modification of the surfaces of a gasactivated carbon and a chemically activated carbon with nitric acid, hypochlorite, and ammonia," Carbon, vol. 32, no. 4, pp. 675-686, 1994.
[42] J. M. Solar, C. A. L. y Leon, K. Osseo-Asare, and L. R. Radovic, "On the importance of the electrokinetic properties of carbons for their use as catalyst supports," Carbon, vol. 28, no. 2-3, pp. 369-375, 1990.

[43] J. Regalbuto, Catalyst Preparation: Science and Engineering, Taylor \& Francis, 2006.

[44] J. P. Tessonnier, D. Rosenthal, T. W. Hansen et al., "Analysis of the structure and chemical properties of some commercial carbon nanostructures," Carbon, vol. 47, no. 7, pp. 1779-1798, 2009 . 

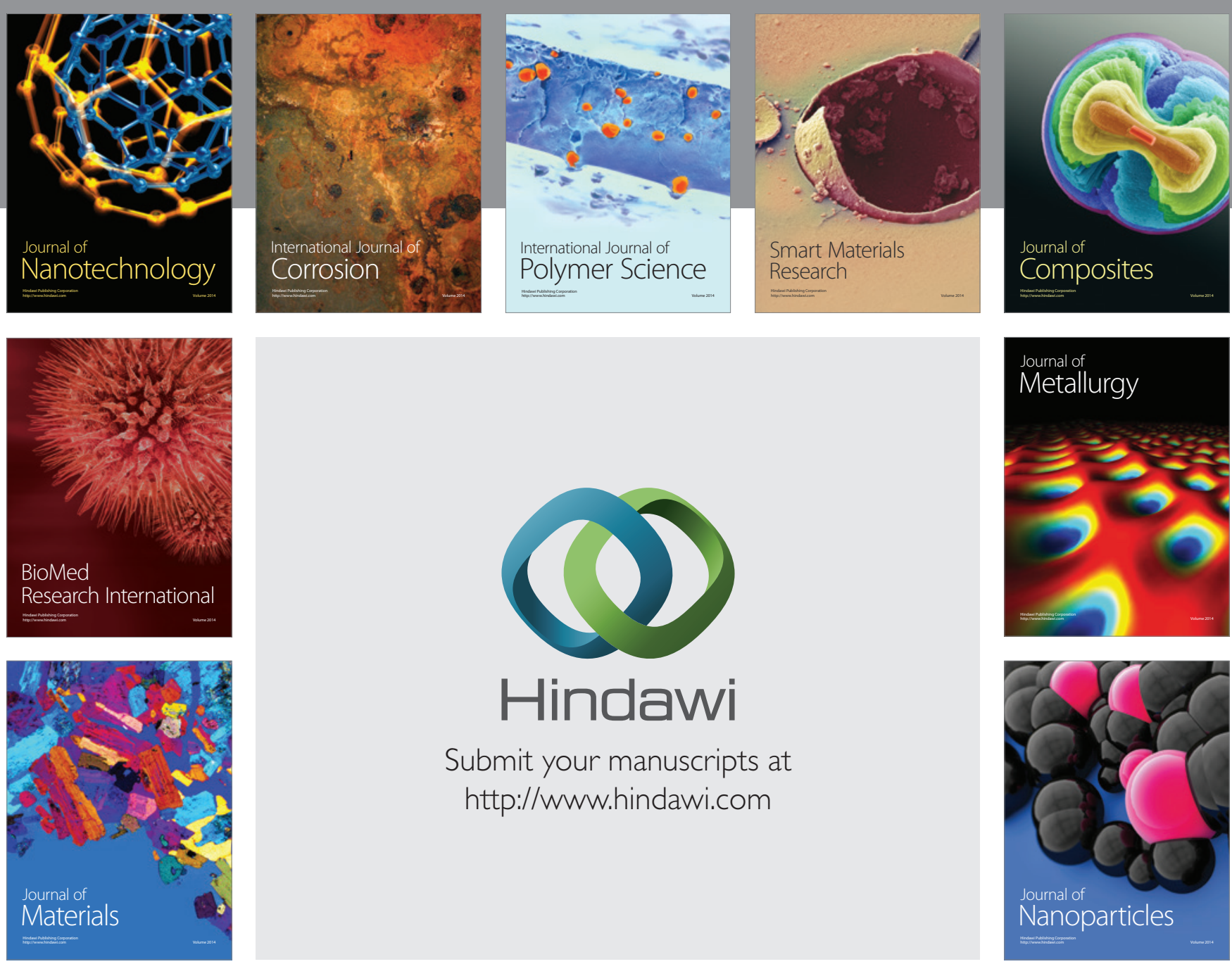

Submit your manuscripts at http://www.hindawi.com
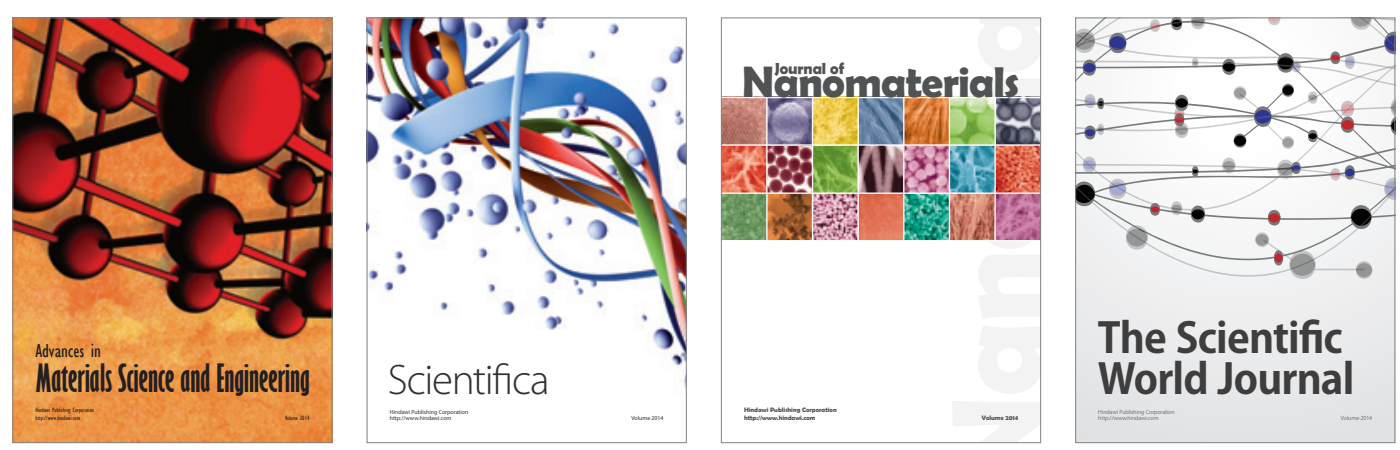

\section{The Scientific World Journal}
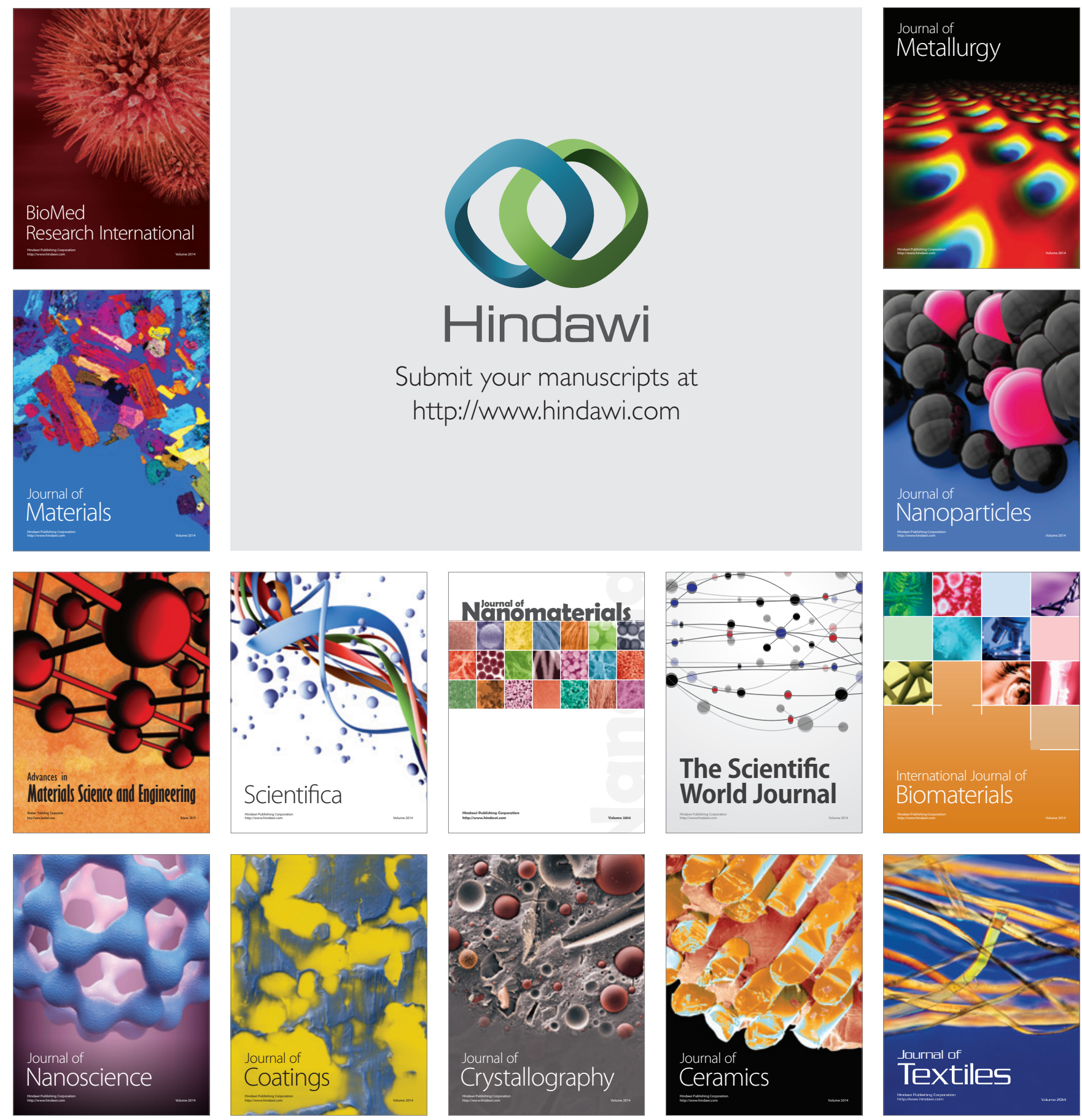